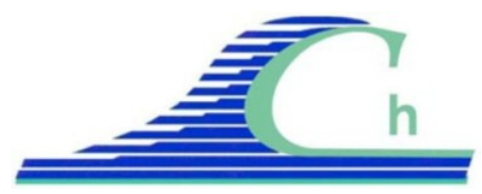

XII ${ }^{\text {ìmes }}$ Journées Nationales Génie Côtier - Génie Civil

Cherbourg, 12-14 juin 2012

DOI:10.5150/jngcgc.2012.048-S @ Editions Paralia CFL

disponible en ligne - http://www.paralia.fr - available online

\title{
Dynamique des suspensions estuariennes en présence de mascarets (cas de la Garonne)
}

\section{Aldo SOTTOLICHIO ${ }^{1}$, Adrien CRAPOULET ${ }^{1,2}$, Jean-Paul PARISOT ${ }^{1}$, Natalie BONNETON $^{1}$, Philippe BONNETON $^{1}$, Guillaume DETANDT ${ }^{1}$}

1. Université Bordeaux 1, Laboratoire EPOC, UMR 5805 CNRS, avenue des Facultés, 33405 Talence, France.

a.sottolichio@epoc.u-bordeaux1.fr

2. Université du Littoral Côte d’Opale, UMR LOG 8187, MREI2, 189A avenue

Maurice Schumann, 59140 Dunkerque, France. Adrien.Crapoulet@univ-littoral.fr

\section{Résumé :}

Le mascaret est ressaut de marée remontant les estuaires, se propageant vers l'amont après la renverse de marée basse. Le but de cette étude est de quantifier les sédiments en suspension en présence de mascarets dans l'estuaire de la Gironde. Pour cela, la turbidité a été enregistrée à $140 \mathrm{~km}$ en amont de l'embouchure, à deux périodes contrastées de débit fluvial. En étiage, la concentration au fond atteint plusieurs dizaines de $\mathrm{g}^{-1}$; les grands mascarets provoquent l'érosion des sédiments et un renversement brutal du flux de MES vers l'amont, 30 fois supérieur au flux de la fin du jusant. Toutefois, ce flux reste faible en comparaison à celui advecté depuis l'aval durant le reste du flot. Le débit fluvial, par son effet sur la position du bouchon vaseux, contrôle le stock de sédiments fins et l'impact du mascaret sur la concentration en MES.

\section{Mots-clés :}

Mascaret - Turbidité Optique - Estuaire de la Gironde - Flux de MES - crème de vase

\section{Abstract:}

Tidal bores are peculiar waves that propagate upwards in shallow estuaries, after low tide reversal. The aim of this study is to quantify suspended sediment transport in the presence of such bores in the upper Gironde estuary. Turbidity was recorded at $140 \mathrm{~km}$ upstream from the mouth, for two contrasted river flow regimes. Results show that during the dry season, near-bed concentration reach levels of fluid mud. The highest tidal bores generate intense resuspension and an upward suspended sediment flux, 30 times greater than that of the final ebb. However, the bore-induced fluxes are weak in comparison with later advection by flood tidal currents. The river discharge, because its effect on the turbidity maximum, controls mean turbidity in the upper estuary, and therefore the relative impact of tidal bores on fine sediment transport.

\section{Introduction}

Le mascaret, appelé "tidal bore" en anglais, est une onde positive de translation qui se forme dans certains estuaires, lorsque les conditions tidales et de débit fluvial sont 
favorables (CHANSON, 2004). Cette onde se caractérise par une série de vagues se propageant vers l'amont à la renverse de marée, juste après l'étale de basse mer. Le phénomène implique l'interaction complexe entre la propagation de la marée en milieu peu profond, la genèse et la propagation d'un ressaut hydraulique et la morphologie des fonds. En général, un mascaret peut être déferlant ou ondulé ; c’est ce dernier type qui est observé dans le système Gironde-Garonne-Dordogne. Des études antérieures, menées dans d'autres environnements, ont montré que le passage du mascaret provoque un affouillement des fonds vaseux, une remise en suspension de ces sédiments fins ainsi que leur transport vers l'amont (TESSIER \& TERWINDT, 1994 ; CHEN et al., 1990 ; BARTSCH-WINCKLER \& LYNCH, 1988). Ces informations sont surtout qualitatives. Peu ou pas de données quantitatives existaient sur le réel impact du mascaret sur la dynamique sédimentaire, et les premières mesures fines sont très récentes (CHANSON et al., 2011). L'objectif de ce travail est d'effectuer une première analyse de mesures originales de turbidité en présence de mascarets, et de discuter l'impact de ces derniers sur les flux sédimentaires estuariens.

\section{Matériel et méthodes}

Les données obtenues proviennent de deux campagnes réalisées dans la Garonne amont. La première au eu lieu de février à avril 2010, sous des débits fluviaux élevés (entre 650 et $750 \mathrm{~m}^{3} \mathrm{~s}^{-1}$ ). La seconde campagne s'est déroulée entre septembre et octobre 2010, sous des débits moyens d'étiage (entre 80 et $170 \mathrm{~m}^{3} \mathrm{~s}^{-1}$ ). La zone d'étude est située face à Podensac, à $140 \mathrm{~km}$ en amont de l'embouchure (figure 1). Cette zone correspond à une portion de section rectiligne du fleuve, favorable au développement de gros mascarets sur toute la largeur, et sans perturbations dues aux méandres. De plus, cet endroit est relativement peu fréquenté par les surfeurs, ce qui réduit les risques d'accident avec le matériel de mesures.

Lors des deux campagnes, un dispositif instrumental conséquent a été déployé, avec un réseau de courantomètres ADCP et des capteurs de pression, dont l'analyse a pour but d'étudier à haute résolution la propagation de l'onde et les vitesses associées (BONNETON et al., 2011a; 2011b). Dans le présent travail, nous exploitons des données relatives à la turbidité, obtenues à partir d'une structure benthique pouvant accueillir des sondes autonomes (SOTTOLICHIO et al., 2010). La structure a été installée sur le bord du chenal principal, à proximité d'un ADCP. Pour la campagne de février 2010, la structure contenait un ADV Nortek à 37,5 cm au-dessus du fond, qui enregistrait à $32 \mathrm{~Hz}$. Pour la campagne de septembre 2010, à côté de l'ADV, un turbidimètre OBS-3A a été installé, pouvant enregistrer à $0,33 \mathrm{~Hz}$. Un deuxième ADV a été posé à $75 \mathrm{~cm}$ du fond, qui enregistrait à $1 \mathrm{~Hz}$. 


\section{XII ${ }^{\text {èmes }}$ Journées Nationales Génie Côtier - Génie Civil \\ Cherbourg, 12-14 juin 2012}
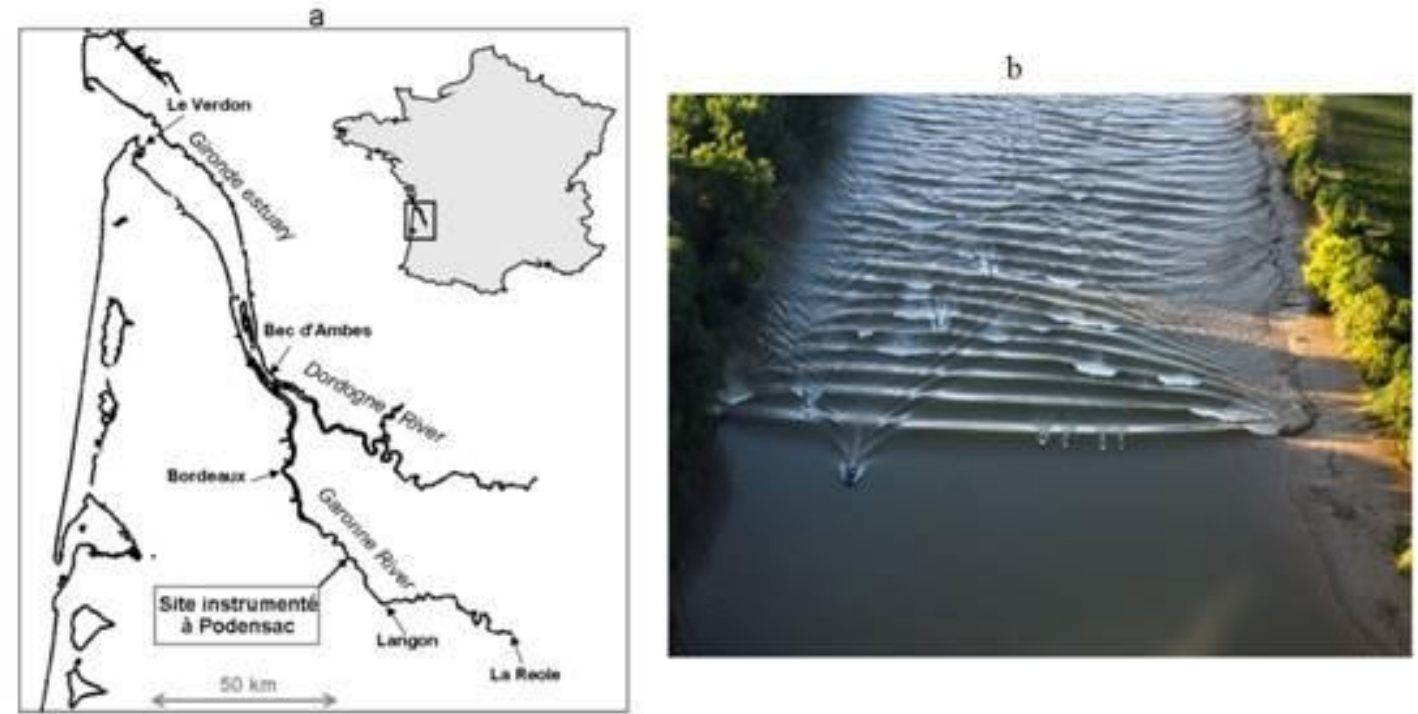

Figure 1. a) carte du système Gironde-Garonne-Dordogne et localisation du site instrumenté de Podensac. b) photographie aérienne du mascaret à Podensac le 10 septembre 2010, 17h31 TU

\section{Détermination de la concentration près du fond}

Les concentrations en matières en suspension (MES) sont obtenues par la calibration des ADV et du turbidimètre OBS. Celle-ci a été réalisée au laboratoire, avec de la vase naturelle de la Garonne diluée progressivement dans l'eau douce. Ainsi, on couvre la gamme de concentrations du milieu naturel, qui peut aller de $0,1 \mathrm{~g} \mathrm{l}^{-1}$ à plusieurs dizaines de $\mathrm{g}^{-1}$. La figure 2 montre la courbe de calibration du turbidimètre OBS-3A. La partie 1 (losanges bleus) est le domaine où le signal reçu par la sonde augmente avec la concentration ; la partie 2 (carrés rouges) est le domaine où la trop forte concentration atténue le signal, qui devient de plus en plus faible. La bascule entre les deux domaines se situe à environ $20 \mathrm{~g} \mathrm{l}^{-1}$. L'exploitation de la partie 2 de la courbe est utile pour détecter la présence éventuelle de couches très concentrées sur le fond (KINEKE et STERNBERG, 1992). Pour l'OBS, nous avons obtenu deux régressions indiquées sur la figure 2, reliant la turbidité $T_{b}$ mesurée par la sonde (en NTU) et la concentration massique $\mathrm{C}_{\mathrm{MES}}$ (en $\mathrm{g} \mathrm{l}^{-1}$ ). Des régressions du même type sont obtenues avec l'amplitude acoustique mesurée par les ADVs (voir un exemple dans SOTTOLICHIO et al., 2010). La conversion des turbidités en concentration en MES requiert de faire le bon choix de l'équation de régression. Pour cela il faut savoir à tout moment si, à signal brut égal, la mesure est effectuée dans un milieu peu concentré ou très concentré. Lors de la campagne de février 2010, les eaux de la Garonne étaient très peu turbides, et les concentrations étaient toujours dans la partie 1 de la calibration $\left(\mathrm{C}_{\mathrm{MES}}<0.5 \mathrm{~g} . \mathrm{l}^{-1}\right)$. En revanche lors de la campagne de septembre 2010, les eaux étaient très turbides et le choix de la régression était plus délicat. Dans ce cas, une information complémentaire 
plus près de la surface s'avère nécessaire, afin de vérifier que les concentrations calculées au fond sont bien supérieures à celles au-dessus. Ceci a été fait à l'aide des deux ADV présents sur la structure. La figure 3 montre une série temporelle de turbidité obtenue par l'OBS (a) et le résultat de l'inversion (b). Autour des marées basses (zones blanches), les concentrations sont généralement faibles, et l'équation 1 a été utilisée. Après le début du flot, la turbidité monte jusqu'à son maximum (4000 NTU) puis chute jusqu'à un minimum à pleine mer. Dans cette zone (en gris) c'est l'équation 2 qui a été utilisée, car elle donne des concentrations cohérentes avec celles des ADV, bien que très fortes. Ceci indique qu'à l'approche de la pleine mer, les concentrations au fond ont continué à augmenter pour atteindre et dépasser $100 \mathrm{~g} \mathrm{l}^{-1}$.

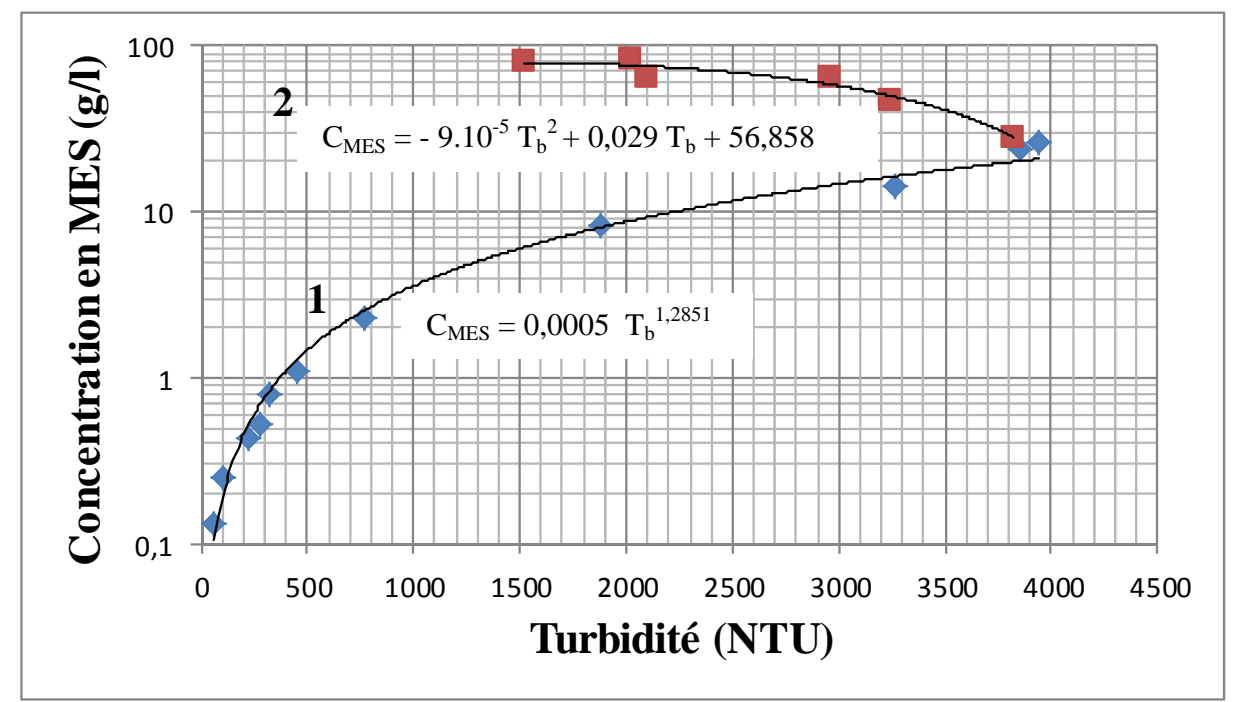

Figure 2. Calibration du turbidimètre OBS 3A 471.
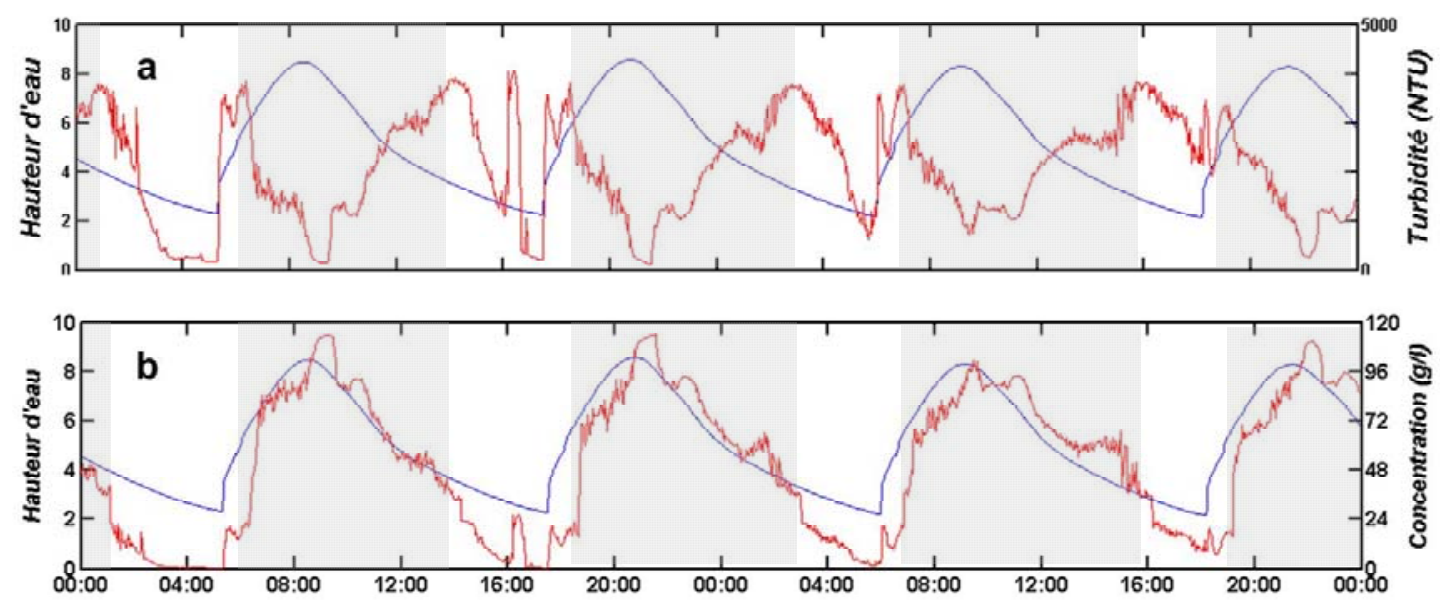

Figure 3. a) Turbidité enregistrée par la sonde OBS $3 A$ à $37 \mathrm{~cm}$ au-dessus du fond, les 9 et 10.09.2010 à Podensac. b) Concentration calculée à partir de la calibration. Les zones grises indiquent le domaine de forte concentration où la courbe 2 a été appliquée. 


\section{XII ${ }^{\text {èmes }}$ Journées Nationales Génie Côtier - Génie Civil \\ Cherbourg, 12-14 juin 2012}

\section{Résultats et discussion}

4.1. Evolution de la concentration au cours d'une marée avec mascaret, en étiage

La figure 4 montre l'évolution conjointe de la hauteur d'eau, du courant et de $\mathrm{C}_{\mathrm{MES}}$ audessus du fond, pour une marée de vive-eau en étiage. Les valeurs sont moyennées sur 1 minute. Le courant descendant est d'environ $1 \mathrm{~m} \mathrm{~s}^{-1}$ à la fin du jusant. La renverse est très rapide, avec un courant qui s’inverse et qui atteint $2 \mathrm{~m} \mathrm{~s}^{-1}$, avent de décélérer progressivement jusqu'à la fin du flot. L'effet du mascaret sur les MES est marqué par un pic de $\mathrm{C}_{\text {MES }}$ au moment de la renverse. En quelques minutes, $\mathrm{C}_{\text {MES }}$ passe de 1 à $20 \mathrm{~g} \mathrm{l}^{-1}$. Le pic est maximum avant le pic de vitesse, indiquant une remise en suspension au passage du train de vagues. Ensuite, après plusieurs minutes, $\mathrm{C}_{\mathrm{MES}}$ continue à augmenter, pour atteindre $60 \mathrm{~g} \mathrm{l}^{-1}$ à mi-flot, et plus de $100 \mathrm{~g} \mathrm{l}^{-1}$ autour de la pleine mer. Ces valeurs sont très élevées et, à l'approche de la pleine mer, on ne voit pas l'effet de la décantation, contrairement à WOLANSKI et al. (2004) dans l'estuaire Daly (Autralie). Ceci serait dû à l'advection d'eau très turbide depuis l'aval de la Garonne, où le noyau du bouchon vaseux se positionne habituellement en période estivale.

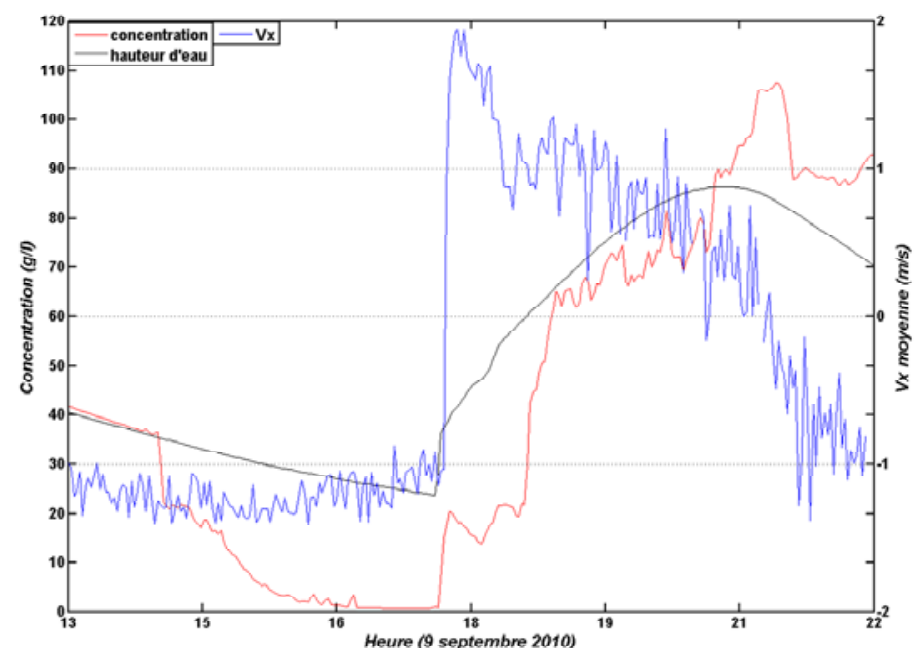

Figure 4. Evolution de la hauteur d'eau, le courant et la concentration en MES audessus du fond sur le site de Podensac le 9 septembre 2010.

Plus récemment, CHANSON et al. (2011), ont mesuré la turbulence au passage du mascaret, en Garonne aval, et rapportent des $\mathrm{C}_{\mathrm{MES}}$ moyennes de $60 \mathrm{~g} \mathrm{l}^{-1}$ autour de la basse mer. Grâce à l'enregistrement du cycle de marée complet, nous montrons que, plus en amont, $\mathrm{C}_{\mathrm{MES}}$ est encore plus élevée après le passage du mascaret, pouvant atteindre des valeurs typiques de la "crème de vase" de la Gironde à la pleine mer. 


\subsection{Effet saisonnier sur l'impact du mascaret}

Le résultat présenté en section 4.1 décrit la situation des grandes marées d'étiage, favorables aux plus forts mascarets. L'examen d'une situation à fort débit fluvial permet d'évaluer l'effet relatif du mascaret en fonction du stock sédimentaire dans le fleuve.
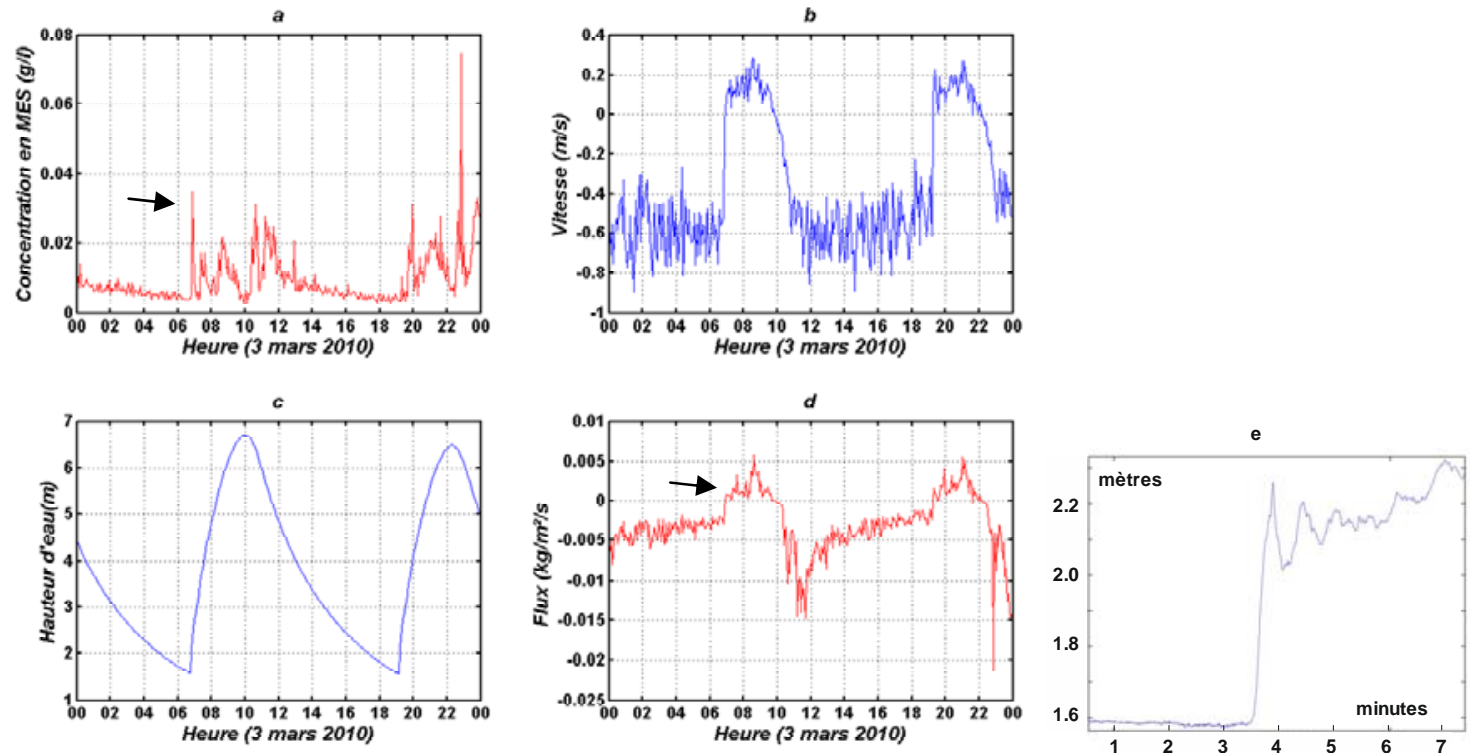

Figure 5. Marée du 03/03/2010 (fort débit fluvial). a) $C_{\text {MES }}$ près du fond, b) vitesse du courant, c) hauteur d'eau, d) flux horizontal de MES. e) zoom autour de la marée basse. La flèche indique le moment du passage du mascaret.
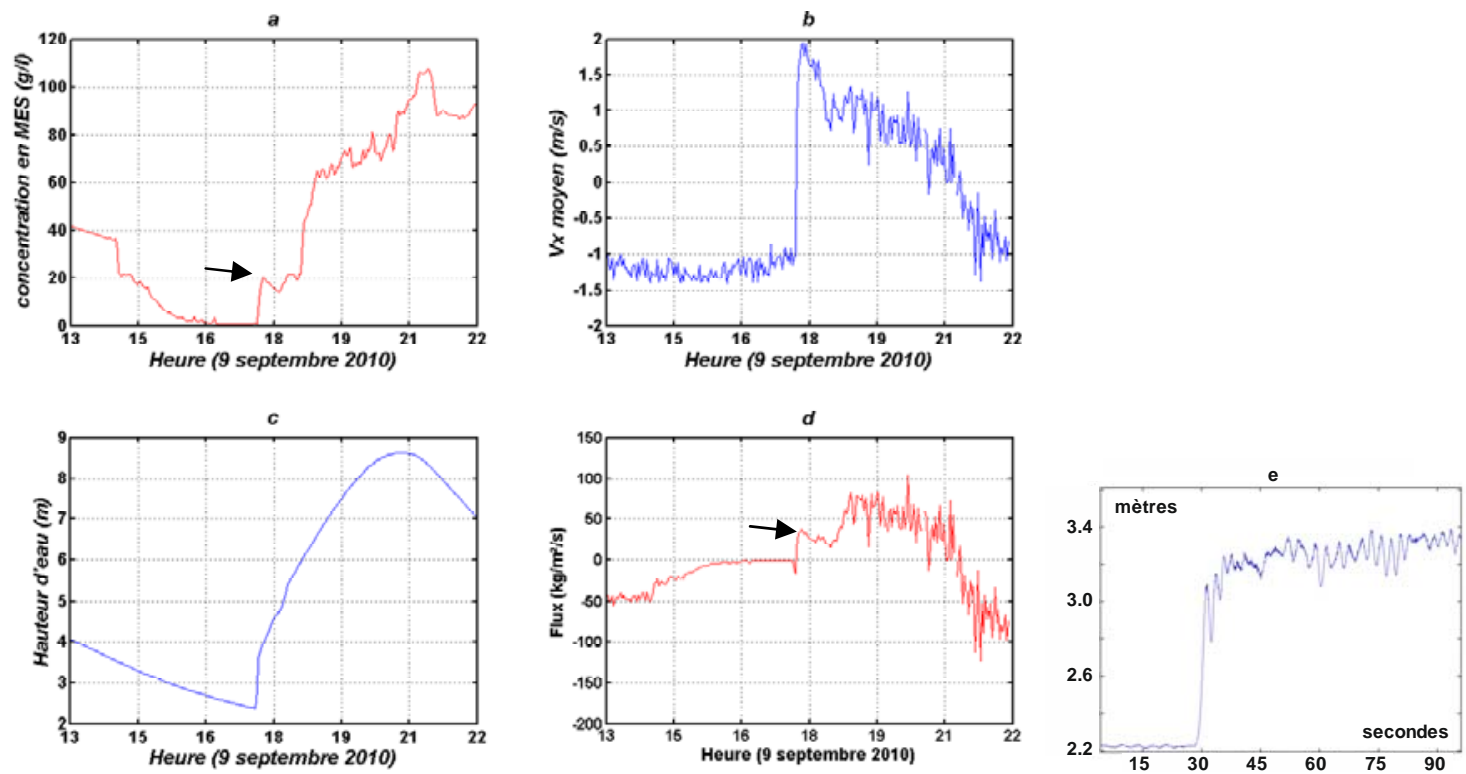

Figure 6. Marée du 09/09/2010 (faible débit fluvial). a) concentration en MES près du fond, b) vitesse du courant, c) hauteur d'eau, d) flux horizontal de MES. e) zoom sur la renverse de marée basse. La flèche indique le moment du passage du mascaret. 


\section{XII ${ }^{\text {èmes }}$ Journées Nationales Génie Côtier - Génie Civil \\ Cherbourg, 12-14 juin 2012}

La figure 5a montre l'évolution de turbidités au cours d'un cycle de marée enregistré lors de la campagne de mars 2010. Malgré le fort débit, un fort mascaret se développe (figure 5e). A cette époque de l'année, le bouchon vaseux est en aval de l'estuaire, et la turbidité en amont est en générale très faible. En effet, $C_{M E S}$ est inférieur à $0,02 \mathrm{~g} \mathrm{l}^{-1}$ pratiquement pendant tout le cycle de marée, sauf lors de pics de concentration, dont un lors du passage du mascaret. Au cours du premier cycle, $\mathrm{C}_{\mathrm{MES}}$ est la plus élevée au passage du mascaret, environ $0,035 \mathrm{~g} \mathrm{l}^{-1}$ (flèche sur la figure $5 \mathrm{a}$ ). Les vitesses de courants sont faibles (jamais supérieures à $0,8 \mathrm{~m} \mathrm{~s}^{-1}$ ), et à dominante de jusant (figure 5b). Les flux de MES calculés sont donc faibles, et dirigés majoritairement vers l'aval (figure 5d). Le flux généré par le mascaret est ici peu significatif en regard des valeurs enregistrées avant et après.

La figure 6 montre l'évolution des paramètres lors de la marée du 9 septembre 2010, situation déjà illustré en figure 4. La présence du bouchon vaseux en amont de l'estuaire génère de plus fortes concentrations. L'effet du mascaret engendre une concentration de $20 \mathrm{~g} \mathrm{l}^{-1}$, concentration relativement peu élevée par rapport à celle enregistrée en fin de flot, comme commenté précédemment. Cependant, cette concentration intervient au maximum de courant ; la contribution du mascaret au flux global de MES advecté vers l'amont en flot est donc plus significative en situation d'étiage.

\section{Conclusions}

Ces premiers résultats indiquent que les gammes de concentration en MES en Garonne amont en étiage sont particulièrement élevées, ce qui est dû à la remontée estivale du bouchon vaseux dans les fleuves. L'enregistrement de la turbidité est délicat dans cet environnement, et nécessite une interprétation minutieuse des conditions de mesure des sondes, qu'elles soient optiques ou acoustiques. La superposition de plusieurs points de mesures sur la verticale s'avère indispensable pour lever les ambigüités près du fond.

Les enregistrements de la turbidité présentés ici ont été obtenus sur plusieurs marées consécutives, pendant tout le cycle, et pour des conditions hydrologiques saisonnières contrastées. Ceci permet de resituer l'importance relative du mascaret sur les flux de MES dans cette partie de l'estuaire.

En période de forts débits fluviaux, il est important de souligner qu'un ressaut de marée peut se développer, ce qui n’avait jamais été documenté avant nos mesures. Dans ces conditions, le flux de MES est majoritairement dirigé vers l'aval. L'effet du mascaret sur l'érosion est visible et contribue significativement à l'augmentation de la turbidité de l'eau, qui est globalement faible $\left(<0,5 \mathrm{~g} \mathrm{l}^{-1}\right)$. Cependant le flux de MES vers l'amont est faible, et la contribution du mascaret est peu importante.

En période d'étiage, l'effet de la prédominance du courant de flot vers l'amont est accentué, et la présence du bouchon vaseux engendre des concentrations globalement très élevées (plusieurs dizaines de $\mathrm{g} \mathrm{l}^{-1}$ ). L'effet du mascaret, est relativement moins 
prépondérant dans l'augmentation de la concentration au cours du cycle de marée. Mais sa contribution au flux global de MES vers l'amont semble plus significative. Le mascaret est surtout un facteur de remise en suspension de sédiment (CHANSON et al., 2011) ; sa contribution à la contrainte de cisaillement sur le fond totale doit encore être évaluée, par une analyse approfondie du jeu de données hydrosédimentaires obtenu lors des campagnes de mesure, en particulier aux échelles de la turbulence.

\section{Références bibliographiques}

BARTSCH-WINKLER S., LYNCH B. (1988). Catalogue of Worldwide Tidal Bore Occurrences and Characteristics. US Geological Survey Circular 1022

BONNETON P., VAN DE LOOCK J., PARISOT J.P., BONNETON N., SOTTOLICHIO A., DETANDT G., CASTELlE B., MARIEU V., POCHON N. (2011a). On the occurrence of tidal bores - The Garonne River case. Journal of Coastal Research, SI 64, 1462-1466.

BONNETON N., BONNETON P., PARISOT J.P., DETANDT G., SOTTOLICHIO A., CRAPOULET A. (2011b). Structure verticale des courants associés à la propagation de la marée dans la Garonne - Impact du mascaret. Proceedings 20 ème Congrès Français de Mécanique, Août 2011, Besançon.

CHANSON H. (2004). The Hydraulics of Open Channel Flows: An Introduction. Butterworth-Heinemann, Oxford, UK, 2nd edition, 630 p.

CHANSON H., REUNGOAT D., SIMON B., LUBIN P. (2011). High-frequency turbulence and suspended sediment concentration measurements in the Garonne River tidal bore. Estuarine, Coastal and Shelf Science, 95, pp 298-306. doi:10.1016/j.ecss.2011.09.012

CHEN J., LIU C., ZHANG C., WALKER H.-J. (1990). Geomorphological Development and Sedimentation in Qiantang Estuary and Hangzhou Bay. Journal of Coastal Research., Vol. 6, n³, pp 559-572.

KINEKE G., STERNBERG R. (1992). Measurements of high concentration suspended sediments using the Optical Backscatterance Sensor. Marine Geology 108:253-258

SOTTOLICHIO A., HURTHER D., LAGUIONIE P., POULLAIN C. (2010). Concentration en matières en suspension dans la crème de vase estuarienne par mesures optiques et acoustiques. XI ${ }^{\text {èmes }}$ Journées Nationales Génie Côtier - Génie Civil, Les Sables d'Olonne, 22-25 juin, pp 557-564. doi:10.5150/jngcgc.2010.065-S

TESSIER B., TERWINDT H.J. (1994). An example of soft sediment deformations in an intertidal environment: the effect of a tidal bore. Comptes Rendus Académie des Sciences, Serie II, 319, pp 217-223.

WOLANSKI E., WILLIAMS D., SPAGNOL S., CHANSON H. (2004). Undular tidal bore dynamics in the Daly Estuary, Northern Australia. Estuarine Coastal and Shelf Science, 60 4, pp 629-636. doi:10.1016/j.ecss.2004.03.001 